\title{
A WHITNEY STRATIFICATION AND EQUISINGULAR FAMILY OF QUASI-ORDINARY SINGULARITIES
}

\author{
CHUNSHENG BAN \\ (Communicated by Louis J. Ratliff, Jr.)
}

\begin{abstract}
Let $(V, 0) \subset\left(C^{d+1}, 0\right)$ be a quasi-ordinary singularity and $\pi$ : $(V, 0) \rightarrow\left(\mathbf{C}^{d}, 0\right)$ a quasi-ordinary projection. $\mathbf{C}^{d}$ has a natural Whitney stratification given by the multiplicities of the discriminant locus of $\pi$. It is proved that the pullback of this stratification gives a Whitney stratification of $(V, 0)$. Then using this result, an equisingular family of quasi-ordinary singularities is studied.
\end{abstract}

\section{WHITNEY STRATIFICATION}

Let $(V, 0)$ be an irreducible hypersurface germ in $\left(\mathbf{C}^{d+1}, 0\right)$ and assume that $0 \in V$ is a singular point (which is not isolated in general). $(V, 0)$ is quasi-ordinary if there is a finite map of analytic germs $\pi:(V, 0) \rightarrow\left(\mathbf{C}^{d}, 0\right)$ that induces a surjection of Zariski tangent spaces and whose discriminant locus $D$ (the hypersurface in $\mathbf{C}^{d}$ over which $\pi$ ramifies) has a normal crossing. $\pi$ is called a quasi-ordinary projection for $(V, 0)$. We may choose local coordinates $x_{1}, x_{2}, \ldots, x_{d}, z$ such that $\pi\left(x_{1}, \ldots, x_{d}, z\right)=\left(x_{1}, \ldots, x_{d}\right)$ and $(V, 0) \subset$ $\left(\mathbf{C}^{d+1}, 0\right)$ is defined by a pseudopolynomial $f$ in $Z$ :

$$
f(Z)=Z^{m}+g_{1}\left(X_{1}, \ldots, X_{d}\right) Z^{m-1}+\cdots+g_{m}\left(X_{1}, \ldots, X_{d}\right)
$$

where the $g_{i}$ are power series in $X_{1}, \ldots, X_{d}$ and $g_{i}(0, \ldots, 0)=0 .(V, 0)$ being quasi-ordinary means that the discriminant $\Delta$ of $f$ has the form

$$
\Delta=X_{1}^{k_{1}} \ldots X_{d}^{k_{d}} u\left(X_{1}, \ldots, X_{d}\right), \quad u(0, \ldots, 0) \neq 0 .
$$

It is known that the roots of $f(Z)=0$ can be represented by fractional power series (cf. [A])

$$
\zeta_{i}=H_{i}\left(X_{1}^{1 / n}, \ldots, X_{d}^{1 / n}\right)=H\left(\omega_{i 1} X_{1}^{1 / n}, \ldots, \omega_{i d} X_{d}^{1 / n}\right), \quad 1 \leq i \leq m,
$$

where $H$ is a power series and the $\omega_{i j}$ are $n$th roots of unity. Then we have a parametrization of $(V, 0)$ by $\zeta=H\left(X_{1}^{1 / n}, \ldots, X_{d}^{1 / n}\right)$, i.e., $(V, 0)$ is the

Received by the editors February 13, 1991 and, in revised form, June 7, 1991. The contents of this paper were presented to the Algebraic Geometry Special Session at the AMS meeting in Fargo, ND, on October 25, 1991.

1991 Mathematics Subject Classification. Primary 14B05, 32S05, 32S15; Secondary 14B07, $32 \mathrm{~S} 30$.

Key words and phrases. Quasi-ordinary singularity, discriminant locus, characteristic monomial, equisingularity. 
image of the map $\Phi: U \rightarrow \mathbf{C}^{d+1}$ ( $U$ some neighborhood of 0 in $\left.\mathbf{C}^{d}\right)$ given by

$$
\Phi\left(x_{1}, \ldots, x_{d}\right)=\left(x_{1}^{n}, \ldots, x_{d}^{n}, H\left(x_{1}, \ldots, x_{d}\right)\right) .
$$

$\zeta$ is called a quasi-ordinary parametrization of $(V, 0)$.

Since the discriminant is $\Delta=\prod_{i \neq j}\left(\zeta_{i}-\zeta_{j}\right)$, unique factorization of (fractional) power series gives

$$
\zeta_{i}-\zeta_{j}=X_{1}^{a_{1} / n} \cdots X_{d}^{a_{d} / n} u_{i j}\left(X_{1}^{1 / n}, \ldots, X_{d}^{1 / n}\right)
$$

where $u_{i j}(0, \ldots, 0) \neq 0$ and $a_{l}$ depends on $i, j \quad(1 \leq l \leq d)$.

The fractional monomials $M_{i j}=X_{1}^{a_{1} / n} \cdots X_{d}^{a_{d} / n}$ so obtained are called the characteristic monomials of $\zeta=H\left(X_{1}^{1 / n}, \ldots, X_{d}^{1 / n}\right)$. This set of monomials determines quite a lot of geometry and topology of $(V, 0)$ (cf. [B, G, L1, L2]).

Let $\pi:(V, 0) \rightarrow\left(C^{d}, 0\right)$ and $D$ be as before and $|D|$ be the reduced discriminant locus of $\pi$. Then $\mathbf{C}^{d}$ has a filtration

$$
D_{0}=\mathbf{C}^{d} \supset D_{1}=|D| \supset D_{2} \supset \cdots
$$

where $D_{i}=\left\{x \in \mathbf{C}^{d} \mid \mu_{|D|}(x) \geq i\right\}$ and $\mu_{|D|}(x)$ is the multiplicity of $|D|$ at $x$.

Proposition 1. The stratification of $\mathbf{C}^{d}$ associated to the above filtration is a Whitney stratification.

Proof. Left to the reader. Q.E.D.

Relabeling the variables if necessary, we may assume that the discriminant $\Delta$ of $f(Z)$ is divisible by $X_{i}$ if and only if $i \leq c$. Then $|D|$ is defined by $X_{1} \cdots X_{c}=0$ and $\left(x_{1}, \ldots, x_{d}\right) \in D_{i}$ if and only if at least $i$ of $x_{1}, \ldots, x_{c}$ are 0 . Let

$$
A_{I}=\left\{\left(x_{1}, \ldots, x_{d}\right) \in \mathbf{C}^{d} \mid x_{i}=0 \text { for } i \in I\right\}, \quad I \subset\{1,2, \ldots, c\},
$$

and $A_{I}^{\circ}=A_{I}-\bigcup_{I \subset J} A_{J}$. Then the connected components of $D_{i}-D_{i+1}$ are the $A_{I}^{\circ}$ with $I \subset\{1,2, \ldots, c\}$ and $|I|=i$. Let $V_{I}^{\circ}=\pi^{-1}\left(A_{I}^{\circ}\right)$ and $V_{I}=\pi^{-1}\left(A_{I}\right)$ be the pullbacks of $A_{I}^{\circ}$ and $A_{I}$, respectively. Since $\pi \mid V_{I}^{\circ}: V_{I}^{\circ} \rightarrow A_{I}^{\circ}$ is an étale covering (cf. [L2, Proposition 7.1.2, p. 86]), $V=\bigcup V_{I}^{\circ}$ is a complex analytic stratification. The following theorem says it is also a Whitney stratification.

Theorem 2. $V=\bigcup V_{I}^{\circ}$ is a Whitney stratification.

Proof. Let $V_{I}^{\circ}, V_{J}^{\circ}$ be two strata such that $V_{I}^{\circ} \subset \overline{V_{J}^{\circ}}=V_{J}$ and $p \in V_{I}^{\circ}$. To show that $\left(V_{J}^{\circ}, V_{I}^{\circ}\right)$ satisfies Whitney conditions at $p$, we begin with some reduction.

By the characterization of the characteristic monomials given by Lipman (cf. [L2, Proposition 1.5]), $V_{J}$ is a quasi-ordinary singularity with quasi-ordinary parametrization $\zeta_{I}$, where $\zeta_{I}$ is the fractional power series obtained from $\zeta$ by substituting 0 for every one of the $X_{i}, i \in J$. So we may assume that $J=\varnothing$, i.e., $V_{J}=V$. Without loss of generality, we may assume that $I=$ $\{1,2, \ldots, e\} \subset\{1,2, \ldots, c\}, e \leq c$. So we have to check the Whitney conditions for $\left(V_{J}^{\circ}, V_{I}^{\circ}\right)=\left(V^{\circ}, V_{\{1,2, \ldots, e\}}^{\circ}\right)$ at $p \in V_{\{1,2, \ldots, e\}}^{\circ}$ where $V^{\circ}=V_{\varnothing}^{\circ}$.

By a lemma of Lipman and its proof (cf. [L2, Lemma 7.2.3]), there exists an analytic automorphism $\psi:\left(\mathbf{C}^{d+1}, p\right) \rightarrow\left(\mathbf{C}^{d+1}, 0\right)$ that maps $(V, p)$ onto 
a subgerm $\left(V^{\prime}, 0\right)$ of $\left(\mathbf{C}^{d+1}, 0\right)$ such that $\left(V^{\prime}, 0\right)$ has quasi-ordinary parametrization

$$
\zeta^{\prime}=H^{\prime}\left(X_{1}^{1 / n}, \ldots, X_{e}^{1 / n}, X_{e+1}, \ldots, X_{d}\right)
$$

that has no characteristic monomial (in this case, $\left(V^{\prime}, 0\right)$ is smooth) or whose smallest characteristic monomial has the form

$$
X_{1}^{a_{1} / n} \cdots X_{e}^{a_{e} / n}, \quad a_{i} \text { integers. }
$$

Under this automorphism, $\left(V_{\{1,2, \ldots, e\}}, p\right)$ is mapped onto a subgerm $(Y, 0)$ of $\left(V^{\prime}, 0\right)$ where $Y$ is defined by $X_{1}=\cdots=X_{e}=0$ in $\left(V^{\prime}, 0\right)$. Then $\zeta^{\prime}$ can be written as

$$
\zeta^{\prime}=X_{1}^{a_{1} / n} \ldots X_{e}^{a_{e} / n} \varepsilon\left(X_{1}^{1 / n}, \ldots, X_{d}\right)+H_{0}\left(X_{1}, \ldots, X_{d}\right)
$$

where $\varepsilon(0, \ldots, 0) \neq 0$ and $H_{0}\left(X_{1}, \ldots, X_{d}\right)$ is the sum of all those terms $c X_{1}^{\rho_{1}} \cdots X_{d}^{\rho_{d}}(c \neq 0)$ appearing in $\zeta^{\prime}$ for which every exponent $\rho_{i}$ is an integer. By the analytic automorphism

$$
\left(x_{1}, \ldots, x_{d}, z\right) \rightarrow\left(x_{1}, \ldots, x_{d}, z-H_{0}\left(x_{1}, \ldots, x_{d}\right)\right)
$$

of the germ $\left(\mathbf{C}^{d+1}, 0\right),\left(V^{\prime}, 0\right)$ is mapped onto a quasi-ordinary hypersurface germ with quasi-ordinary parametrization

$$
\zeta^{\prime}-H_{0}=X_{1}^{a_{1} / n} \ldots X_{e}^{a_{e} / n} \varepsilon\left(X_{1}^{1 / n}, \ldots, X_{d}\right), \quad \varepsilon(0, \ldots, 0) \neq 0 .
$$

Therefore, it suffices to check the Whitney conditions for $\left(V^{\circ}, Y\right)$ at $0 \in Y$ where $V$ has a quasi-ordinary parametrization

$$
\zeta=X_{1}^{a_{1} / n} \ldots X_{e}^{a_{e} / n} \varepsilon\left(X_{1}^{1 / n}, \ldots, X_{d}\right), \quad \varepsilon(0, \ldots, 0) \neq 0 .
$$

Then $(*)$ can be written as

$(* *) \quad \Phi\left(x_{1}, \ldots, x_{d}\right)=\left(x_{1}^{n}, \ldots, x_{e}^{n}, x_{e+1}, \ldots, x_{d}, x_{1}^{a_{1}} \ldots x_{e}^{a_{e}} \varepsilon\left(x_{1}, \ldots, x_{d}\right)\right)$

and $V^{\circ}=V_{\varnothing}^{\circ}=\left\{\left(x_{1}, \ldots, x_{d}, z\right) \in V \mid x_{1}, \ldots, x_{e} \neq 0\right\}$. Let

$$
P_{k}=\Phi\left(x_{k 1}, \ldots, x_{k d}\right) \in V^{\circ}, \quad Q_{k}=\left(0, \ldots, 0, y_{k e+1}, \ldots, y_{k d}, 0\right) \in Y,
$$

such that $P_{k} \rightarrow 0, Q_{k} \rightarrow 0$. Suppose $T_{P_{k}}=T_{P_{k}} V \rightarrow T$ and $P_{k} Q_{k} \rightarrow L=$ $\left(L_{1}: \cdots: L_{d+1}\right)$. We need to show that $L \subset T$.

By abuse of notation, we also use $T_{P_{k}}, T$ to indicate the direction of $T_{P_{k}}$, $T$ respectively.

The parametrization $(* *)$ gives $T_{P_{k}}=\left((-1)^{1} M_{1}, \ldots,(-1)^{d+1} M_{d+1}\right)$ where $M_{i}$ is the minor of the Jacobian matrix $D \Phi\left(P_{k}\right)$ with the $i$ th column deleted. So we have

$$
\begin{aligned}
T_{P_{i}}=\left(\frac{x_{k}^{a}}{n x_{k 1}^{n}}\left(a_{1} \varepsilon+x_{k 1} \frac{\partial \varepsilon}{\partial x_{1}}\right), \ldots, \frac{x_{k}^{a}}{n x_{k e}^{n}}\left(a_{e} \varepsilon+x_{k e} \frac{\partial \varepsilon}{\partial x_{e}}\right),\right. \\
\left.\quad x_{k}^{a} \frac{\partial \varepsilon}{\partial x_{e+1}}, \ldots, x_{k}^{a} \frac{\partial \varepsilon}{\partial x_{d}},-1\right)
\end{aligned}
$$

where $x_{k}^{a}=x_{k 1}^{a_{1}} \cdots x_{k e}^{a_{e}}$. The secant $P_{k} Q_{k}$ is given by

$$
P_{k} Q_{k}=\left(x_{k 1}^{n}, \ldots, x_{k e}^{n}, x_{k e+1}-y_{k e+1}, \ldots, x_{k d}-y_{k d}, x_{k} a_{\varepsilon}\right) \text {. }
$$

Write $T=\left(b_{1}, \ldots, b_{d+1}\right)$. Then $T_{P_{k}} \rightarrow T$ and $P_{k} Q_{k} \rightarrow L$ mean that there exist $\lambda_{k}, \mu_{k} \in \mathbf{C}$ such that

$$
\lim \lambda_{k} T_{P_{k}}=T, \quad \lim \mu_{k} P_{k} Q_{k}=L .
$$


Writing the two limits componentwise, we get

$$
\begin{aligned}
& \lim \lambda_{k} \frac{x_{k}^{a}}{n x_{k j}^{n}}\left(a_{j} \varepsilon+x_{k j} \frac{\partial \varepsilon}{\partial x_{j}}\right)=b_{j}, \quad 1 \leq j \leq e, \\
& \lim \lambda_{k} x_{k}^{a} \frac{\partial \varepsilon}{\partial x_{j}}=b_{j}, \quad e<j \leq d, \\
& \lim \left(-\lambda_{k}\right)=b_{d+1}, \\
& \lim \mu_{k} x_{k j}^{n}=L_{j}, \quad 1 \leq j \leq e, \\
& \lim \mu_{k}\left(x_{k j}-y_{k j}\right)=L_{j}, \quad e<j \leq d, \\
& \lim \mu_{k} x_{k}^{a} \varepsilon=L_{d+1} .
\end{aligned}
$$

Then

$$
\begin{aligned}
T \cdot L= & b_{1} L_{1}+\cdots+b_{d+1} L_{d+1}=\lim \lambda_{k} T_{P_{k}} \cdot \mu_{k} P_{k} Q_{k} \\
= & \lim \left(\sum_{j=1}^{e} \frac{\lambda_{k} \mu_{k} x_{k}^{a}}{n}\left(a_{j} \varepsilon+x_{k j} \frac{\partial \varepsilon}{\partial x_{j}}\right)\right. \\
& \left.\quad+\sum_{j=e+1}^{d} \lambda_{k} \mu_{k} x_{k}^{a}\left(x_{k j}-y_{k j}\right) \frac{\partial \varepsilon}{\partial x_{j}}-\lambda_{k} \mu_{k} x_{k}^{a} \varepsilon\right) \\
= & -b_{d+1} L_{d+1}\left(\sum_{j=1}^{e} \frac{a_{j}}{n}-1\right) .
\end{aligned}
$$

If $b_{d+1} L_{d+1}=0$, then $T \cdot L=0$. If $b_{d+1} L_{d+1} \neq 0$, then we may assume that $b_{d+1}=-1, L_{d+1}=\varepsilon(0)$ and take $\lambda_{k}=1, \mu_{k}=1 / x_{k}^{a}$. Then (1) and (1') show that both $\operatorname{limits} \lim x_{k}^{a} / x_{k j}^{n}$ and $\lim x_{k j}^{n} / x_{k}^{a}$ exist for $1 \leq j \leq e$, and so the $\operatorname{limits} \lim \left(x_{k}^{a} / x_{k j}^{n}\right)^{a_{j}}$ and $\lim \left(x_{k j}^{n} / x_{k}^{a}\right)^{a_{j}}$ exist for $1 \leq j \leq e$. Then the limits

$$
\begin{aligned}
& \lim \prod_{j=1}^{e}\left(\frac{x_{k}^{a}}{x_{k j}^{n}}\right)^{a_{j}}=\lim \left(x_{k}^{a}\right)^{\left(a_{1}+\cdots+a_{e}\right)-n}, \\
& \lim \prod_{j=1}^{e}\left(\frac{x_{k j}^{n}}{x_{k}^{a}}\right)^{a_{j}}=\lim \left(x_{k}^{a}\right)^{n-\left(a_{1}+\cdots+a_{e}\right)}
\end{aligned}
$$

exist. Since $\lim x_{k}^{a}=0$, we must have $n-\left(a_{1}+\cdots+a_{e}\right)=0$, i.e., $\sum_{j=1}^{e} a_{j} / n-1$ $=0$. We still have $T \cdot L=0$ in (4). Therefore $L \subset T$. This completes the proof. Q.E.D.

\section{EQUISINGULAR FAMILY}

As an application, we now study the equisingularity of a family of quasiordinary singularities.

Let $\left\{\left(V_{t}, 0_{t}\right) \mid t \in S\right\}$ be a family of $d$-dimensional hypersurface germs parametrized by a smooth germ $(S, s)$ of dimension $r$. Let $V$ be the total space and $W$ be the locus of the origins $0_{t}$. Namely, there is a flat morphism $\phi:(V, 0) \rightarrow(S, s)$, where $0=0_{s}$, of complex analytic germs and a section $i:(S, s) \rightarrow(V, 0)$ of $\phi$ such that $V_{t}=\phi^{-1}(t)$ and $i(t)=0_{t}$. Then $W=i(S)$ 
is a smooth subspace of $V$ that is isomorphic to $S$. We have the following notions of equisingularity (cf. [Z]):

- $V$ is topologically equisingular along $W$ at 0 if all $\left(V_{t}, 0_{t}\right)$ have the same topological type for $t$ close to $s$, where we say that two germs $\left(V_{1}, 0_{1}\right)$ and $\left(V_{2}, 0_{2}\right)$ in $\left(\mathbf{C}^{d+1}, 0\right)$ have the same topological type if there exists a local homeomorphism $\rho:\left(\mathbf{C}^{d+1}, V_{1}, 0_{1}\right) \rightarrow\left(\mathbf{C}^{d+1}, V_{2}, 0_{2}\right)$ of the triples.

- $V$ is differentially equisingular along $W$ at 0 if $(V, W)$ satisfies the Whitney conditions at 0 .

Now, we assume that there is a finite map $p: V \rightarrow \mathbf{C}^{d} \times S$ such that $p(W)=$ $\{0\} \times S$ is an isomorphism and $p_{t}=p \mid V_{t}: V_{t} \rightarrow \mathbf{C}^{d}$ is a quasi-ordinary projection of $\left(V_{t}, 0_{t}\right)$ for every $t \in S$. Thus $\left\{\left(V_{t}, 0_{t}\right)\right\}$ is a family of quasi-ordinary singularities. Then by the results of Gau and Lipman (cf. [G, L2]), we have

Theorem 3. $V$ is topologically equisingular along $W$ at each point of $W$ if all $\left(V_{t}, 0_{t}\right)$ have the same characteristic monomials. Conversely, $\left(V_{t}, 0_{t}\right)$ have the same (normalized) characteristic monomials (may not be with respect to the $p_{t}$ ) if $V$ is topologically equisingular along $W$.

Therefore, the characteristic monomials determine the topological equisingularity of the family $\left\{\left(V_{t}, 0_{t}\right)\right\}$. Similar results are also true for differential equisingularity. We will prove the following theorems.

Theorem 4. If all $\left(V_{t}, 0_{t}\right)$ have the same characteristic monomials then $W$ is a stratum of a Whitney stratification of $(V, 0)$, and so $(V, 0)$ is differentially equisingular along $W$ at any point of $W$.

Theorem 5. If $W$ is a stratum of a Whitney stratification of $(V, 0)$ then all the $\left(V_{t}, 0_{t}\right)$ have the same (normalized) characteristic monomials.

Proof of Theorem 5. By a well-known result of Thom and Mather, $(V, 0)$ is topologically equisingular along $W$ at each point of $W$. Then all $\left(V_{t}, 0_{t}\right)$ have the same topological type in $\left(\mathbf{C}^{d+1}, 0\right)$. By Theorem 2 , all $\left(V_{t}, 0_{t}\right)$ have the same (normalized) characteristic monomials. Q.E.D.

To prove Theorem 4, we start with some preparation.

Lemma 6. Let $F:(X, x) \rightarrow(S, s)$ be a deformation of $\left(X_{0}, x_{0}\right)$ with a section $\sigma: S \rightarrow X$, and let $X_{t}=F^{-1}(t)$ and $x_{t}=\sigma^{-1}(t)$. If $\left(X_{t}, x_{t}\right)$ is analytically isomorphic to $\left(X_{0}, x_{0}\right)$ for all $t \in(S, s)$, then $F$ is analytically trivial, i.e., $X$ is isomorphic to $\left(X_{0}, x_{0}\right) \times(S, s)$ (cf. [T, Theorem 2.1]).

Since $(S, s)$ is smooth, we may assume that $(S, s)=\left(\mathbf{C}^{r}, 0\right)$. By the flatness of $\phi:(V, 0) \rightarrow\left(\mathbf{C}^{r}, 0\right)$, we may assume that $V \subset \mathbf{C}^{d+1} \times \mathbf{C}^{r}$ is defined by

$$
f\left(X_{1}, \ldots, X_{d+1}, Y_{1}, \ldots, Y_{r}\right)=f(X, Y)=0
$$

and $\phi$ is induced by the second projection $\mathbf{C}^{d+1} \times \mathbf{C}^{r} \rightarrow \mathbf{C}^{r}$. The $\left(V_{t}, 0_{t}\right)$ is defined by $f_{t}(X)=f\left(X_{1}, \ldots, X_{d+1}, t_{1}, \ldots, t_{r}\right)=0$ in $\mathbf{C}^{d+1}$. Since $\left(V_{0}, 0\right)$ is a quasi-ordinary singularity, we may choose the local coordinates $x_{1}, \ldots, x_{d}, z$ for $\mathbf{C}^{d+1}$ at 0 such that

$$
\begin{aligned}
f_{0}\left(X_{1}, \ldots, X_{d}, Z\right) & =f\left(X_{1}, \ldots, X_{d}, Z, 0, \ldots, 0\right) \\
& =Z^{m}+g_{1}\left(X_{1}, \ldots, X_{d}\right) Z^{m-1}+\cdots+g_{m}\left(X_{1}, \ldots, X_{d}\right)
\end{aligned}
$$


as before. Then $f(0, \ldots, 0, Z, 0, \ldots, 0)=Z^{m}$. By the Weierstrass preparation theorem, we may assume

$$
f\left(X_{1}, \ldots, X_{d}, Z, Y_{1}, \ldots, Y_{r}\right)=Z^{m}+a_{1}(X, Y) Z^{m-1}+\cdots+a_{m}(X, Y) .
$$

Also, we may identify $p: V \rightarrow \mathbf{C}^{d} \times S=\mathbf{C}^{d} \times \mathbf{C}^{r}$ with the restriction to $V$ of

$$
\pi \times \mathrm{id}: \mathbf{C}^{d+1} \times \mathbf{C}^{r} \rightarrow \mathbf{C}^{d} \times \mathbf{C}^{r}
$$

where $\pi\left(x_{1}, \ldots, x_{d}, z\right)=\left(x_{1}, \ldots, x_{d}\right)$. Now, we can prove the following key lemma.

Lemma 7. If all $\left(V_{t}, 0_{t}\right)$ have the same characteristic monomials with respect to $p_{t}$, then the total space $(V, 0)$ itself is a quasi-ordinary singularity with the quasi-ordinary projection $p: V \rightarrow \mathbf{C}^{d} \times S$.

Proof. Let $\Delta=\Delta(X, Y)$ be the discriminant of $f$ (as a polynomial in $Z$ ). Then $\Delta_{t}=\Delta(X, t)$ is the discriminant of $f_{t}\left(X_{1}, \ldots, X_{d}, Z\right)$. Since all $\left(V_{t}, 0_{t}\right)$ have the same characteristic monomials, $\Delta_{t}=\Delta_{0} u_{t}$, where $u_{t}$ is unit, for $t \in$ $\left(\mathbf{C}^{r}, 0\right)$; and so the discriminant locus $\left(D_{t}, t\right)$ of $p_{t}$ is analytically isomorphic to $\left(D_{0}, 0\right)$. If $D$ is the disciminant locus of $p$, then Lemma 6 asserts that $p$ is analytically trivial, and so $D \simeq D \times \mathbf{C}^{r}$ is a normal crossing. Therefore, $(V, 0)$ itself is a quasi-ordinary singularity. Q.E.D.

Now, we can prove Theorem 4.

Proof of Theorem 4. By Lemma 7 and its proof, $(V, 0)$ is a quasi-ordinary singularity defined by $f\left(X_{1}, \ldots, X_{d}, Z, Y_{1}, \ldots, Y_{r}\right)=0$, where

$$
f\left(0, \ldots, 0, Y_{1}, \ldots, Y_{r}\right) \equiv 0 \text {, }
$$

with discriminant

$$
\Delta=X_{1}^{a_{1}} \ldots X_{e}^{a_{e}} u\left(X_{1}, \ldots, X_{d}, \ldots, Y_{1}, \ldots, Y_{r}\right), \quad u(0, \ldots, 0) \neq 0 .
$$

Let $V=\bigcup V_{I}^{\circ}$ be the Whitney stratification as in Theorem 2 .

If $e=d$ in (5), $W=0 \times \mathbf{C}^{r}=V_{\{1,2, \ldots, d\}}=V_{\{1,2, \ldots, d\}}^{\circ}$; and so $W$ is a stratum of $V=\bigcup V_{I}^{\circ}$.

If $e<d$ in (5), then by $(* *), V_{\{1,2, \ldots, e\}}^{\circ}=\{0\} \times \mathbf{C}^{d+1-e} \times \mathbf{C}^{r}$ and we have

$$
W=0 \times \mathbf{C}^{r} \subset V_{\{1,2, \ldots, e\}}^{\circ}=\left(0 \times \mathbf{C}^{d+1-e}\right) \times \mathbf{C}^{r} .
$$

It is trivial that $\left(V_{\{1,2, \ldots, e\}}^{\circ}-W, W\right)$ satisfies Whitney conditions. For any stratum $V_{I}^{\circ}$ with $|I|<e$, since $\left(V_{I}^{\circ}, V_{\{1,2, \ldots, e\}}^{\circ}\right)$ satisfies Whitney conditions and $W \subset V_{\{1,2, \ldots, e\}}^{\circ},\left(V_{I}^{\circ}, W\right)$ and $\left(V_{I}^{\circ}, V_{\{1,2, \ldots, e\}}^{\circ}-W\right)$ satisfy Whitney conditions. Then we have a Whitney stratification

$$
V=\left(\bigcup_{|I|<e} V_{I}^{\circ}\right) \cup\left(V_{\{1,2, \ldots, e\}}^{\circ}-W\right) \cup W
$$

with $W$ as a stratum. This completes the proof. Q.E.D.

\section{ACKNOWLEDGMENT}

The author would like to thank Professor Joseph Lipman for his encouragement and support. 


\section{REFERENCES}

[A] S. S. Abhyankar, On the ramification of algebraic functions, Amer. J. Math. 77 (1955), 575-592.

[B] C. Ban, Whitney stratification, equisingular family and the auréole of quasi-ordinary singularity, Thesis, Purdue Univ., 1990.

[G] Y. N. Gau, Embedded topological classification of quasi-ordinary singularities, Mem. Amer. Math. Soc., vol. 74, Amer. Math. Soc., Providence, RI, 1988, pp. 109-129.

[HL] J.-P. G. Henry and D. T. Lé, Limits d'espaces tangents, Lecture Notes in Math., vol. 482, Springer-Verlag, New York, 1975, pp. 251-265.

[L1] J. Lipman, Quasi-ordinary singularities of embedded surfaces, Thesis, Harvard Univ., 1965.

[L2] _ Topological invariants of quasi-ordinary singularities, Mem. Amer. Math. Soc., vol. 74, Amer. Math. Soc., Providence, RI, 1988, pp. 1-107.

[LT] D. T. Lé and B. Teissier, Limits d'espaces tangents en géométrie analytique, Comment. Math. Helv. 63 (1988), 540-578.

[T] B. Teissier, Deformations a type topologique constant. I, Astérisque 16 (1974), 215-249.

[W] H. Whitney, Tangents to an analytic variety, Ann. of Math. (2) 81 (1964), 496-549.

[Z] O. Zariski, Some open questions in the theory of singularity, Bull. Amer. Math. Soc. 77 (1971), 481-491.

Department of Mathematics, Ohio State University, Mansfield, Ohio 44906

E-mail address: cban@mps.ohio-state.edu 